\title{
Management of anesthesia and complications in children with Tracheobronchial Foreign Body Aspiration
}

\author{
Erol Karaaslan ${ }^{1}$, Turan Yildiz ${ }^{2}$
}

\begin{abstract}
Objectives: Delayed diagnosis and treatment of tracheobronchial foreign body aspiration (FBA) in children may lead to morbidity and mortality. Our objective was to evaluate the anesthetic management, periand post-operative complications, and predisposing factors for postoperative intensive care unit (ICU) admission in children undergoing rigid bronchoscopy due to tracheobronchial FBA.

Methods: This retrospective study included 81 children who underwent rigid bronchoscopy between January 2010 to July 2018 at Inonu University, Department of Pediatric Surgery, Turkey. Data regarding demographic characteristics, anesthetic management, length of ICU and hospital stays, and peri- and postoperative complications were retrieved from the hospital database.

Results: The patients included $54(66.7 \%)$ boys and $27(33.3 \%)$ girls with a mean age of $29.6 \pm 31.2$ months. The most common presenting symptom was the suspicion of FBA, followed by acute-onset cough, cyanosis, wheezing and respiratory distress. Mean duration of anesthesia was $44.40 \pm 14.72 \mathrm{~min}$. Of the 81 patients, $18(22.2 \%)$ were transferred to ICU and $63(77.8 \%)$ patients were transferred to the ward postoperatively. Of the patients transferred to the ICU, 5 of them required mechanical ventilation. Some peri and postoperative complications, desaturation $(n=16 ; 19.7 \% ; p=0.001)$, mucosal bleeding $(n=6 ; 7.4 \%$; $p=0.02)$, laryngeal edema $(n=11 ; 13.6 \% ; p<0.001)$, laryngospasm $(n=13 ; 16.3 \% ; p<0.001)$, were affected the frequency of intensive care transfer.

Conclusion: Bronchoscopy with general anesthesia remains the golden standard for the management of tracheobronchial FBA. In such patients, a combination consisting of a detailed preoperative clinical evaluation of the patient, selection of short-acting anesthetic agents with minimal side effects for the induction and maintenance of anesthesia, and the administration of controlled ventilation can be recommended. Additionally, early diagnosis of peri- and post-operative complications, prediction of postoperative ICU requirement, and a close cooperation of anesthesiologists and surgeons are highly important.
\end{abstract}

KEYWORDS: Anesthetic management, Bronchoscopy, Complications, Intensive care, Tracheobronchial foreign body.

doi: https://doi.org/10.12669/pjms.35.6.1225

How to cite this:

Karaaslan E, Yildiz T. Management of anesthesia and complications in children with Tracheobronchial Foreign Body Aspiration. Pak J Med Sci. 2019;35(6):1592-1597. doi: https://doi.org/10.12669/pjms.35.6.1225

This is an Open Access article distributed under the terms of the Creative Commons Attribution License (http://creativecommons.org/licenses/by/3.0), which permits unrestricted use, distribution, and reproduction in any medium, provided the original work is properly cited.

1. Dr. Erol Karaaslan,

Assistant Professor, Dept. of Anesthesiology and Reanimation,

2. Dr. Turan Yildiz,

Associate Professor, Dept. of Pediatric Surgery,

1,2: Inonu University, Medical Faculty,

Malatya, Turkey.

Correspondence:

Dr. Erol Karaaslan,

Assistant Professor, Dept. of Anesthesiology and Reanimation,

Inonu University, Medical Faculty,

Elazig Yolu 15. Km, 44280 Battalgazi, Malatya, Turkey.

E-mail: erol44kara@gmail.com

* Received for Publication:

June 13, 2019

* Accepted for Publication:

Septemebr 19, 2019

\section{INTRODUCTION}

Tracheobronchial foreign body aspiration (FBA) is the aspiration of a foreign body (FB) into the tracheobronchial tree through the mouth or nose during inhalation and is the most common cause of acuteonset upper respiratory airway obstruction. Approximately $80 \%$ of tracheobronchial FBA cases are seen in children aged 0-3 years and tracheobronchial FBA is responsible for $0-1.8 \%$ of deaths in this age group. Moreover, FBA is the most common cause of accidental deaths in children less than one-year-old. ${ }^{1,2}$ 
Bronchoscopy is the standard treatment modality for confirming the presence of a tracheobronchial FB in children and removing it. Rigid bronchoscope is mostly used for the removal of solid FBs. Undiagnosed and retained FBs may lead to serious complications such as pneumonia, lung abscess, and bronchiectasis. ${ }^{2,3}$ Rigid bronchoscopies is safely performed under general anesthesia in an operating room environment. Given the shared use of the airway by the surgeon and the anesthesiologist, bronchoscopy requires experienced teams for securing sufficient ventilation and oxygenation in the patient. ${ }^{4}$ Anesthetic managements during bronchoscopic procedures, particularly the anesthetic technique to be used, is of prime importance due to the risk of peri- and postoperative complications. Successful anesthetic management and removal of an aspirated FB has a contributory effect on the reduction of morbidity and mortality. .5

The aim of this study was to evaluate the demographic characteristics, anesthetic management, periand post-operativecomplications, and predisposing factors for postoperative intensive care unit (ICU) admission in children undergoing rigid bronchoscopy due to tracheobronchial FBA.

\section{METHODS}

This retrospective study included 95 children who were diagnosed with tracheobronchial FBA by a combination of patient history, physical examination, and radiological findings and underwent emergency or elective rigid bronchoscopy in Inonu University, Department of Pediatric Surgery between January 2010 to July 2018. This trial was approved by the Local Ethic Committee of Inonu University (Protocol no: 2019/3-15). Of these, 14 patients were excluded from the study and thus a total of 81 patients were included in the study. The study was conducted according to CONSORT guidance. ${ }^{7}$

The study included pediatric patients aged below 18 years with an American Society of Anesthesiologists (ASA) score of 1-4 who underwent bronchoscopy due to tracheobronchial FBA. Patients that did not undergo preoperative anesthetic evaluation, and had incomplete medical records.

Preoperative Procedures: Premedication was performed with midazolam in all the patients except for the patients with respiratory distress. Following the transfer of the patients to the operating room, standard routine monitoring including noninvasive blood pressure (NIBP), heart rate (HR), peripheral oxygen saturation $\left(\mathrm{SpO}_{2}\right)$, end-tidal carbon dioxide $\left(\mathrm{EtCO}_{2}\right)$, and electrocardiogram was performed.

Following preoxygenation with four $\mathrm{L} /$ minutes $100 \% \mathrm{O}_{2}$ for at least three minutes, general anesthesia was induced with appropriate combinations of propofol (1-2 mg.kg-1) or thiopental sodium (5 mg.kg-1), fentanyl (0.5-1 mcg.kg-1) or remifentanil (0.1-0.25 $\mathrm{mcg} / \mathrm{kg} / \mathrm{min})$, succinylcholine (1 mg.kg-1) or rocuronium $(0.5 \mathrm{mg} / \mathrm{kg})$ at appropriate clinical doses of intravenous (i.v.) anesthetic agents. Anesthetic maintenance was achieved with sevoflurane MAC one in a 50:50 oxygen-air mixture. Reversal of neuromuscular blockade was achieved with neostigmine (0.05), atropine (0.02), or sugammadex (2 mg.kg-1). Intermittent positivepressure ventilation was delivered via a side port of a rigid bronchoscope.

Following the bronchoscopy procedure, the patients were transferred to the post-anesthesia care unit (PACU) with ventilation provided either via bag-valve-mask or insertion of an orotracheal tube, as needed. The patients ventilated via bagvalve-mask were transferred to the ward when they achieved a Modified Aldrete's score of 9 or greater (a score of nine on a 0-12 scale indicates recovery sufficient for the patient to be transferred from PACU to the ward). ${ }^{8}$ The patients with orotracheal intubation were transferred to the ICU. Postoperative analgesia was achieved with paracetamol $(20 \mathrm{mg} / \mathrm{kg}$, i.v) in all the patients.

Demographic characteristics, anesthetic management, presenting symptoms (suspicion of FBA, acute-onset persistent cough, cyanosis, wheezing, respiratory distress, nausea, vomiting, pneumonia), durations of anesthesia and surgery, peri- and post-operative complications (laryngeal edema, laryngospasm, bronchospasm, bradycardia, desaturation, mucosal bleeding, cough, nausea, vomiting, aspiration, cardiac arrest, and death), type and localization of aspirated FBs, length of ICU and hospital stays, and mortality were recorded for each patient.

Duration of anesthesia was defined as the time period from the transfer of the patient to the operating room to the transfer of the patient to PACU. Duration of surgery was defined as the time period from the initial introduction of the rigid bronchoscope tip in the patient's mouth to the removal of the bronchoscope from the vocal cords. Laryngospasm was defined as glottal closure caused by the reactions obstructing ventilation of the lungs. Bronchospasm was defined as a prolonged expiratory phase accompanied by 
wheezing and desaturation. Bradycardia was defined as a resting heart rate of below 60 beats per minute. Desaturation was defined as a $\mathrm{SpO} 2$ level of $94 \%$ or below for more than 15 sec. Mucosal bleeding was defined as the presence of bleeding associated with the bronchoscopy procedure. Length of ICU stay was defined as the time period from the transfer of the patient to the ICU to the transfer of the patient to the ward postoperatively. Length of hospital stay was defined as the time period from hospital admission due to the suspicion of FBA to hospital discharge. Mortality was defined as death occurring in association with anesthesia or surgery during the hospital stay.

Quantitative variables were expressed as mean \pm standard deviation (SD) and qualitative variables were expressed as frequencies and percentages. Qualitative data were analyzed with Yates corrected chi-square or Fisher's exact test as appropriate. $\mathrm{P}<0.05$ values were considered as significant. IBM SPSS Statistics version 25.0 for Windows was used for statistical analysis.

\section{RESULTS}

The 81 patients included $54(66.7 \%)$ boys and 27 $(33.3 \%)$ girls with a mean age of $29.6 \pm 31.2$ months. The most common presenting symptom was the suspicion of FBA, followed by acute-onset cough, cyanosis, wheezing, respiratory distress, nauseavomiting, and pneumonia. The ASA score was I in $18(22.2 \%)$, II in $62(76.5 \%)$, and III in 1 (1.3\%) patient. Mallampati score was I in $71(87.7 \%$ and II in $10(12.3 \%)$ patients (Table-I).

General anesthesia was induced with sodium thiopental in $5(6.1 \%)$, propofol in $76(93.8 \%)$, remifentanil in $64(76 \%)$, and fentanyl in $17(20.7 \%)$ patients. Neuromuscular blockade was achieved with rocuronium in $71(87.7 \%)$ and succinylcholine in $10(12.3 \%)$ patients. Sevoflurane was used for anesthetic maintenance in all the patients $(100 \%)$. Reversal of neuromuscular blockade was achieved with sugammadex in 43 (60.5\%) and with neostigmine-atropinein 28 (39.5\%) patients. Prednol one $\mathrm{mg} / \mathrm{kg}$ was administered in all the patients intraoperatively. Mean duration of anesthesia was $44.40 \pm 14.72 \mathrm{~min}$ and mean duration of surgery was $27.35 \pm 14.20 \mathrm{~min}$. Perioperative complications included desaturation $(\mathrm{n}=16 ; 19.7 \%)$, bradycardia $(\mathrm{n}=8 ; 9.8 \%)$, mucosal bleeding $(\mathrm{n}=6 ; 7.4 \%)$, laryngeal edema $(n=11 ; 13.6 \%)$, laryngospasm $(n=13 ; 16.3 \%)$, and bronchospasm $(\mathrm{n}=4 ; 4.6 \%)$. The incidence of laryngeal edema, laryngospasm, desaturation, and mucosal bleeding was significantly higher in the patients transferred to the ICU postoperatively $(p<0.05)$ (Table-II).

Foreign bodies (FBs) were successfully extracted in $71(87.7 \%)$ patients and no FBs were detected in the remaining $10(12.3 \%)$ patients. The extracted FBs were mostly localized in the right main bronchus $(\mathrm{n}=35 ; 43.3 \%)$, followed by the larynx/trachea $(n=20 ; 24.6 \%)$ and the left main bronchus $(n=16$; $19.7 \%$ ). No patient had a FB lodged in both bronchi.

Of the 81 patients, $18(22.2 \%)$ were transferred to the ICU postoperatively, five of whom required mechanical ventilation. Twenty-five (30.8\%) patients with no acute respiratory distress were pre-medicated with midazolam and eight of them required postoperative ICU admission. Of the 18 patients transferred to the ICU postoperatively, 12 $(66.7 \%)$ patients had organic and $4(22.2 \%)$ patients had inorganic FBs, whereas $2(11.1 \%)$ patients were detected with no FBs. Of the 16 FBs extracted in the patients transferred to the ICU, $8(44.4 \%)$ of them were localized in the right bronchus, 6 $(33.3 \%)$ in the left bronchus, and $2(11.1 \%)$ in the trachea-larynx. Mean length of ICU and hospital stays were $0.65 \pm 1.29$ and $2.25 \pm 1.29$ days for 81 patients, respectively.

Table-I: Demographics and procedure data

\begin{tabular}{lc}
\hline & $n(\%)$ or Mean \pm std \\
\hline Age, month & $29.06 \pm 31.20$ \\
Gender, $\boldsymbol{n}$ & \\
$\quad$ Male & $54(66.7)$ \\
$\quad$ Female & $27(33.3)$ \\
Weight, kg & $25.59 \pm 12.97$ \\
ASA, $\boldsymbol{n}(\%)$ & \\
$\quad$ I & $18(22.2)$ \\
$\quad$ II & $62(76.5)$ \\
III & $1(1.2)$ \\
IV & $0(0)$ \\
Mallampati & \\
$\quad$ I & $71(88.8)$ \\
$\quad$ II & $10(12.3)$ \\
III & $0(\%)$ \\
Duration of Anesthesia, min & $44.30 \pm 14.72$ \\
Duration of Surgery, min & $27,35 \pm 14.20$ \\
Length of Hospital Stay (LOS), day & $2.25 \pm 1.69$ \\
Length of ICU, day & $0.65 \pm 1.29$ \\
Localization of Foreign Body (FB) & \\
$\quad$ FB in right bronchial tree, $\mathrm{n}(\%)$ & $35(43.3)$ \\
$\quad$ FB in left bronchial tree, $\mathrm{n}(\%)$ & $16(19.7)$ \\
$\quad$ FB in larynx and trachea, $\mathrm{n}(\%)$ & $20(24.6)$ \\
$\quad$ No FB & $10(12.3)$ \\
\hline
\end{tabular}

The data is presented as percentage or number of cases n: number of cases. 
Erol Karaaslan et al.

Table-II: Peri- and postoperative complications.

\begin{tabular}{|c|c|c|c|c|c|c|}
\hline \multirow[t]{3}{*}{ Complications } & & \multicolumn{4}{|c|}{ Postoperative service of patients } & \multirow{3}{*}{$P$} \\
\hline & & \multicolumn{2}{|c|}{ Ward } & \multicolumn{2}{|c|}{$I C U$} & \\
\hline & & $n$ & $\%$ & $n$ & $\%$ & \\
\hline \multirow[t]{2}{*}{ Laryngeal edema } & Absent & 60 & 85.7 & 10 & 14.3 & $<0.001^{*}$ \\
\hline & Present & 3 & 27.3 & 8 & 72.7 & \\
\hline \multirow[t]{2}{*}{ Laryngospasm } & Absent & 58 & 86.6 & 9 & 13.4 & $<0.001^{*}$ \\
\hline & Present & 4 & 30.8 & 9 & 69.2 & \\
\hline \multirow[t]{2}{*}{ Bronchospasm } & Absent & 61 & 79.2 & 16 & 20.8 & $0.212^{*}$ \\
\hline & Present & 2 & 50.0 & 2 & 50.0 & \\
\hline \multirow[t]{2}{*}{ Desaturation } & Absent & 56 & 86.2 & 9 & 13.8 & $0.001^{*}$ \\
\hline & Present & 7 & 43.8 & 9 & 56.3 & \\
\hline \multirow[t]{2}{*}{ Mucosal Bleeding } & Absent & 61 & 81.3 & 14 & 18.7 & $0.02^{*}$ \\
\hline & Present & 2 & 33.3 & 4 & 66.7 & \\
\hline \multirow[t]{2}{*}{ Coughing } & Absent & 45 & 83.3 & 9 & 16.7 & $0.156 \#$ \\
\hline & Present & 18 & 66.7 & 9 & 33.3 & \\
\hline \multirow[t]{2}{*}{ Nausea } & Absent & 55 & 77.5 & 16 & 22.5 & $0.61^{*}$ \\
\hline & Present & 8 & 80.0 & 2 & 20.0 & \\
\hline \multirow[t]{2}{*}{ Vomiting } & Absent & 60 & 80.0 & 15 & 20.0 & $0.120^{*}$ \\
\hline & Present & 3 & 50.0 & 3 & 50.0 & \\
\hline \multirow[t]{2}{*}{ Bradycardia } & Absent & 59 & 80.8 & 14 & 19.2 & $0.068^{*}$ \\
\hline & Present & 4 & 50.0 & 4 & 50.0 & \\
\hline \multirow[t]{2}{*}{ Death } & Absent & 63 & 100 & 18 & 100 & \\
\hline & Present & 0 & 0 & 0 & 0 & \\
\hline
\end{tabular}

*: Fisher's exact test; \#: Yates corrected chi-square test.

Of the 81 patients, $63(77.8 \%)$ of them were transferred to the ward postoperatively, among whom supplemental oxygen was administered via nasal cannula in $50(79.4 \%)$, nausea occurred in $10(12.3 \%)$, and vomiting occurred in $6(7.4 \%)$ patients. No signs of aspiration were observed in any patient peri- or post-operatively.

\section{DISCUSSION}

Most of tracheobronchial FBA cases are seen in children aged 1-3 years, predominantly in boys. Children in this age group are particularly susceptible to FBA due to the propensity to insert objects in their mouth, lack of molars leading to ineffective chewing, and their tendency to play, laugh, and move around while eating. ${ }^{5,9,10}$ As consistent with the literature, most of the patients in our study were aged below three years and the incidence of FBA was higher in boys compared to girls.

Literature indicates that there is no consensus on the anesthetic technique to be used in the management of tracheobronchial FBA in children. In such patients, selection of the anesthetic technique and agents is based on the ventilation technique planned for each patient. These ventilation techniques include spontaneous and controlled ventilation, both of which have been shown to have both advantages and disadvantages. Liu et al. compared spontaneous and controlled ventilation and reported that there was no difference between the two techniques with regard to desaturation while the incidence of laryngospasm was higher in the patients that underwent spontaneous ventilation. ${ }^{11}$ However, in a previous retrospective study, Chen et al. reported that spontaneous ventilation led to a greater incidence of intraoperative body movement, straining, laryngospasm, and hypoxia and significantly longer duration of emergence from anesthesia compared to other ventilation techniques. ${ }^{12}$

A controlled ventilation technique, consisting of administration of neuromuscular blockade, has been shown to have several advantages such as prevention of body movement and straining, increased anesthetic depth, easy passage of the bronchoscope through the vocal cords, and reduction of the incidence of airway obstruction and atelectasis. ${ }^{6,13}$ However, controlled ventilation can be disadvantageous as well since it may require additional positive-pressure breaths, thereby 
leading to unintentional movement of the FB further distally. ${ }^{13,14}$ In our study, we also performed controlled positive-pressure ventilation in our patients.

Selection of anesthetic agents for a surgical procedure also depends on the personal knowledge and experience of anesthesiologists, with propofol and sodium thiopental reported to be the most common agents for anesthetic induction. ${ }^{15}$ In our study, propofol was administered in all the patients.

There are numerous studies in the literature reporting on the use and advantages of sevoflurane in the induction and maintenance of anesthesia in children undergoing bronchoscopy due to tracheobronchial FBA. Accordingly, sevoflurane has been shown to be advantageous over other agents since it is relatively less irritant, facilitates the removal of the FB by reducing the airway resistance, and has fewer side effects. ${ }^{16}$ Nevertheless, sevoflurane has been reported to have several disadvantages as well, such as requirement of higher sevoflurane concentrations for the achievement of a deep level of anesthesia during bronchoscopy due to the air leakage around the rigid bronchoscope which leads to pollution of ambient air. ${ }^{17}$ As consistent with the literature, we also used sevoflurane as the inhalation agent in all the patients.

Remifentanil, which is known to have a short half-life, has been shown to be effective in the prevention of hemodynamic response to laryngoscopy. ${ }^{18}$ Additionally, besides its analgesic effects, remifentanil has also been reported to have antitussive effects and favorable effects on cough. ${ }^{19}$ Previous studies reported that patients anesthetized with remifentanil without the administration of neuromuscular blockade had a lower incidence of cough while they had a higher incidence of desaturation and longer duration of emergence from anesthesia. ${ }^{14,20}$ In our study, remifentanil was used in almost all the patients and our findings were consistent with those reported in the literature.

Rocuronium, a non-depolarizing, short-acting neuromuscular blocker with a short half-life, causes minimal hemodynamic response and no histamine release. Rocuronium has been shown to significantly reduce the risk of laryngospasm and bronchospasm when used in combination with remifentanil. ${ }^{21}$ In our study, remifentanil was used in almost all the patients and the findings obtained were consistent with those reported in the literature. Succinylcholine is a depolarizing neuromuscular blocker which is commonly preferred for short surgical procedures, satiated patients, and patients requiring emergency intubation, mainly due to its rapid effects and short half-life. However, when administered in repeated doses, succinylcholine may result in rhabdomyolysis, cardiac arrest, and bradycardia. ${ }^{22}$ Won et al. reported that sugammadex led to faster reversal of rocuronium-induced neuromuscular blockade and shorter extubation time compared to atropine-neostigmine although no significant difference was found with regard to the incidence of adverse events following anesthetic induction. ${ }^{23}$ As consistent with the literature, in our study, reversal of rocuronium-induced neuromuscular blockade was achieved with atropine-neostigmine in 32.1\% and sugammadex in $53.1 \%$ of the patients. On the other hand, succinylcholine was administered in $10(12.3 \%)$ patients that underwent emergency bronchoscopy due to respiratory distress, although all the 10 patients were satiated. Side effects occurred in $5(7.4 \%)$ patients that received rocuronium as opposed to $3(30 \%)$ patients that received succinylcholine. Depending on these findings, it is safe to assert that rocuronium appears to be a better option than succinylcholine in patients planned for elective bronchoscopy under general anesthesia. In addition, no malignant hyperthermia, cardiac arrest, or aspiration occurred in any of our patients.

In a previous retrospective study, Hatipoglu et al. reported that almost $60 \%$ of their patients were transferred to ICU postoperatively and $10 \%$ of these patients required mechanical ventilation. In our study, however, although the need for mechanical ventilation was similar to that of the study by Hatipoglu et al. postoperative ICU admission was relatively lower. ${ }^{4}$ Another study reported that $6(5.8 \%)$ patients required postoperative ICU admission due to sleepiness and sinuous arrhythmia $(\mathrm{n}=1)$, airway edema and the resulting respiratory distress $(\mathrm{n}=2)$, sleepiness and hypoxia $(n=1)$, pneumonia and elongated aspiration $(n=1)$, and respiratory distress and cyanosis $(n=1){ }^{10}$ Williams et al. reported that delayed or missed treatment of desaturation, brachycardia, and bronchospasm may result in serious complications such as laryngeal edema, pneumothorax, and cardiac arrest. In patients with tracheobronchial FBA, steroids are recommended for the treatment of laryngeal edema. ${ }^{24}$ In our study, $18(22.2 \%)$ were transferred to ICU and $63(77.8 \%)$ patients were transferred to the ward postoperatively. Despite not being a cohort study, our study revealed that the incidence of laryngeal edema, laryngospasm, desaturation, 
and mucosal bleeding was significantly higher in the patients transferred to the ICU compared to those transferred to the ward $(p<0.05)$. The rate of mortality associated with tracheobronchial FBA has been shown to be $0-1.8 \% .^{2}$ However, no mortality occurred in our patients.

Limitations of the study: The study had a retrospective design. Further large-scale studies are needed to investigate the predisposing factors for postoperative ICU requirement in patients with tracheobronchial FBA.

\section{CONCLUSION}

Bronchoscopy with general anesthesia remains the golden standard for the management of children with tracheobronchial FBA. We think that selection of short-acting anesthetic agents with minimal side effects for the induction and maintenance of anesthesia, and the administration of controlled ventilation can be recommended in children. Additionally, diagnosis of peri and post-operative complications, prediction of postoperative ICU requirement, and a close cooperation of anesthesiologists and surgeons are highly important.

\section{Source of funding: None.}

Conflicts of interest: None.

\section{REFERENCES}

1. Akcora B, Celikkaya ME, Ozer C. Bronchoscopy for foreign body aspiration and effects of nebulized albuterol and budesonide combination. Pak J Med Sci. 2017;33(1):81-85. doi: 10.12669/pims.331. 11297.

2. Zhao Z, Gao Q, Song P. A rare case of bilateral bronchial foreign body. Pak J Med Sci. 2015;31(2):477-479. doi: 10.12669/ pims.312.6060.

3. Divarci E, Toker B, Dokumcu Z, Musayev A, Ozcan C, Erdener $\mathrm{A}$. The multivariate analysis of indications of rigid bronchoscopy in suspected foreign bodyaspiration. Int J Pediatr Otorhinolaryngol. 2017;100:232-237. doi: 10.1016/j. ijporl.2017.07.012.

4. Hatipoglu Z, Onder O, Ozcengiz D. Anesthesia for rigid bronchoscopy in children: Single center experience. Cukurova Med J. 2018;43(3):678-684. doi: 10.17826/ cumj.337581.

5. Kendigelen $P$. The anaesthetic consideration of tracheobronchial foreign body aspiration in children. J Thorac Dis. 2016;8:38033807. doi: $10.21037 /$ jtd.2016.12.69.

6. Chai J, Wu XY, Han N, Wang LY, Chen WM. A retrospective study of anesthesia during rigid bronchoscopy for airway foreign body removal in children: Propofol and sevoflurane with spontaneous ventilation. Pediatr Anesth. 2014;24:10311036. doi: $10.1111 /$ pan.12509.

7. Schulz KF, Altman DG, Moher D, CONSORT Group. CONSORT 2010 statement: Updated guidelines for reporting parallel group randomised trials. Int J Surg. 2011;9:672-677. doi: 10.1016/j.ijsu.2011.09.004.

8. Aldrete JA, Kroulik DA. A postanesthetic recovery score. Anesth Analg. 1970;49:924-934.
9. Haddadi S, Marzban S, Nemati S, Ranjbar Kiakelayeh S, Parvizi A, Heidarzadeh A. Tracheobronchial Foreign-Bodies in Children; A 7 Year Retrospective Study. Iran J Otorhinolaryngol. 2015;27:377-385.

10. Pekcan S, Aslan AT. Cocukluk caginda yabanci cisim aspirasyonlari. Turkish J Pediatr Dis. 2010;4:119-128.

11. Liu Y, Chen L, Li S. Controlled ventilation or spontaneous respiration in anesthesia for tracheobronchial foreign body removal: A metaanalysis. Pediatr Anesth. 2014;24:1023-1030. doi: $10.1111 /$ pan.12469.

12. Chen LH, Zhang $X$, Li SQ, Liu YQ, Zhang TY, Wu JZ. The risk factors for hypoxemia in children younger than 5 years old undergoing rigid bronchoscopy for foreign body removal. Anesth Analg. 2009;109:1079-1084. doi: 10.1213/ ane.0b013e3181b12cb5.

13. Zur KB, Litman RS. Pediatric airway foreign body retrieval surgical and anesthetic perspectives. Paediatr Anaesth. 2009;19:109-117. doi: 10.1111/j.1460-9592.2009.03006.x.

14. Soodan A, Pawar D. Subramanium R. Anesthesia for removal of inhaled foreign bodies in children. Paediatr Anesth. 2014;14:947952. doi: 10.1111/j.1460-9592.2004.01309.x.

15. Ansermino JM, Magruder W, Dosani M. Spontaneous respiration during intravenous anesthesia in children Curr Opin Anaesthesiol. 2009;22:383-387. doi: 10.1097/ ACO.0b013e328329730c

16. Farrell PT. Rigid bronchoscopy for foreign body removal: Anaesthesia and ventilation. Paediatr Anaesth. 2014;14:84-89. doi: 10.1046/j.1460-9592.2003.01194.x.

17. Meretoja OA, Taivainen T, Raiha L, Korpela R, Wirtavuori K. Sevoflurane-nitrous oxide or halothane-nitrous oxide for paediatric bronchoscopy and gastroscopy. Br J Anaesth. 1996;76:767-777. doi: 10.1093/bja/76.6.767.

18. Ozcan S, Basar H, Anbarci O. Comparison of the effects of Remifentanil and Fentanyl on the hemodynamic response to Tracheal Intubatıon. Turkiye Klinikleri J Med Sci. 2003;23:204.

19. Teksan L, Baris S, Karakaya D, Dilek A. A dose study of remifentanil in combination with propofol during tracheobronchial foreign bodyremoval in children. J Clin Anesth. 2013;25:198-201. doi: 10.1016/j.jclinane.2012.10.008.

20. Liao R, Li JY, Liu GY. Comparison of sevoflurane volatile induction/maintenance anaesthesia and propofol-remifentanil total intravenous anaesthesia for rigid bronchoscopy under spontaneous breathing for tracheal/bronchial foreign body removal in children. Eur J Anaesthesiol. 2010;27:930-934. doi: 10.1097/EJA.0b013e32833d69ad.

21. Qiu Y, Qu J, Li X, Li H. Anesthesia with propofol-remifentanil combined with rocuronium for bronchial foreign body removal in children: Experience of 2886 cases. Pediatr Invest. 2018;2:2529. doi: $10.1002 /$ ped 4.12030 .

22. Orebaugh SL. Succinylcholine: adverse effects and alternatives in emergency medicine. Am J Emerg Med. 1999;17:715-721. doi: 10.1016/S0735-6757(99)90168-7.

23. Won YJ, Lim BG, Lee DK, Kim H, Kong MH, Lee IO. Sugammadex for reversal of rocuronium-induced neuromuscular blockade in pediatric patients: A systematic review and meta-analysis. Medicine (Baltimore) 2016;95:4678. doi: 10.1097/MD.0000000000004678.

24. Williams A, George C, Atul PS, Sam S, Shukla S. An audit of morbidity and mortality associated with foreign body aspiration in children from a tertiary level hospital in Northern India. Afr J Paediatr Surg. 2014;11:287-292. doi: 10.4103/0189-6725.143129.

\section{Authors' Contribution:}

EK: Study design, data collection and analysis, manuscript preparation and drafting.

TY: Study design, Review and final approval of manuscript. 